\title{
A Nonlinear Exact Disturbance Observer Inspired by Sliding Mode Techniques
}

\author{
Xinkai Chen \\ Department of Electronic Information Systems, Shibaura Institute of Technology, 307 Fukasaku, Minuma-ku, Saitama-shi, \\ Saitama Prefecture 337-8570, Japan \\ Correspondence should be addressed to Xinkai Chen; chen@shibaura-it.ac.jp
}

Received 25 May 2014; Accepted 21 July 2014

Academic Editor: Ligang Wu

Copyright (c) 2015 Xinkai Chen. This is an open access article distributed under the Creative Commons Attribution License, which permits unrestricted use, distribution, and reproduction in any medium, provided the original work is properly cited.

Inspired by sliding mode techniques, a nonlinear exact disturbance observer is proposed. The disturbance and its derivatives up to the second order are assumed to be bounded. However, the bounds of the disturbance and its derivatives are unknown, and they are adaptively estimated online during the observation of the disturbances. The exact convergence of the disturbance observer to the genuine disturbance is assured theoretically. The convergence speed of the disturbance estimation error is controlled by design parameters. The proposed method is robust to the type of disturbance and is easy to be implemented. Computer simulation results show the superiority and effectiveness of the proposed formulation.

\section{Introduction}

In all practical control systems, the existence of unknown disturbances, model uncertainties, and uncertain nonlinearities is inevitable. In the past decades, disturbance observation has been one of the major topics in control engineering. The motivation is suggested by the fact that if the disturbances can be estimated, then control of the uncertain dynamic systems with disturbances may become easier. For example, the controller with disturbance cancellation functions can be easily constructed by using the estimated disturbances [1-6]. The disturbance observer approaches in the literature have been developed either in the transfer function domain or in the state space domain.

Due to the intuitive and simple logic of the disturbance observers based on the inversion of transfer function, it has been applied in the tracking controllers for practical motion control systems [7-9]. In this kind of design, an "inner loop" around the controlled system is closed in order to reject the disturbances and to urge the relation between the input and the output to approximate the so-called "nominal model" of the controlled system at low frequency domain. The inner loop is tuned by adjusting a low pass filter. One typical shortcoming of this formulation is that the control performance is not satisfactory when the type of the disturbance is unknown and there exist unmodeled dynamics [10].

Another approach which is based on the state space domain is also extensively studied in the literature due to its allowance of transient behavior analysis [11-15]. Among the many presented methods, a typical formulation of estimating the disturbance which is based on the variable structure system (VSS) techniques is proposed in [14]. By estimating the unknown states and their derivatives by using the inputoutput information, eventually, the disturbances in the state space expression are estimated. One shortcoming of this kind of formulation is that a high order observer structure needs to be constructed.

From the robustness point of view, the advantages of variable structure control system are well addressed in [1618]. Thus, by nature, the VSS theory is to be applied to the robust disturbance observer formulation. In the literature, it is well known that, for minimum phase dynamical systems with relative degree one, the disturbance can be estimated by using the VSS "equivalent control theory" $[17,18]$. However, this approach cannot cope with systems with higher relative degrees. Furthermore, the VSS equivalent control approach is not theoretically strict since, on the sliding surface $S(t)=0$, 
the derivative of $S(t)$ cannot be proved to be zero. To overcome these difficulties, approximate nonlinear disturbance observers are proposed in $[19,20]$ for systems with arbitrary relative degrees, where strict analysis has been achieved for the disturbance estimating precision.

In this paper, inspired by the sliding mode control theory, a nonlinear exact disturbance observer is proposed based on state space approach. The "disturbance" may be the model uncertainties, the external disturbance, the combination of them, and so forth. The disturbance and its derivatives up to the second order are assumed to be bounded. Since these bounds are usually unknown in practice, a priori information of the value of the bounds is not required. In the disturbance estimating process, the bounds relating to the disturbances are simultaneously estimated online by using adaptive method. The contribution of the paper is that an exact asymptotic estimation of the disturbance can be achieved theoretically, whereas only approximate estimate could be obtained in the literature $[1,7-11,13,19,20]$. Convergence of the proposed observer is strictly analyzed and proved for arbitrary initial conditions of the disturbance observer. The convergence speed of the disturbance estimation error is controlled by design parameters. The proposed disturbance observer is robust to the types of the disturbances $[7,8,10]$ and is easy to be implemented. Comparison with a nonlinear disturbance observer in the literature [19] is conducted, where the transient time and the robustness to measurement noises are considered. Computer simulation results illustrate the effectiveness and superiority of the proposed formulation.

\section{Problem Statement}

Consider an uncertain system of the form

$$
\dot{x}(t)=f(x(t), u(t), t)+d(t),
$$

where $x(t)=\left[x_{1}(t), \ldots, x_{n}(t)\right]^{T} \in R^{n}$ is the state; $u(t) \epsilon$ $R$ is the input; $f(x(t), u(t), t) \in R^{n}$ is a known function of $t, x(t)$, and $u(t) ; d(t)=\left[d_{1}(t), \ldots, d_{n}(t)\right]^{T} \in R^{n}$ is an unknown vector which is composed of external disturbances, model uncertainties, and so forth. For simplicity, $d(t)$ is called "disturbance" in the following part of this paper.

This paper tries to propose an exact nonlinear method to estimate the disturbance $d(t)$. For this purpose, the following two assumptions are made.

(A1) The state $x(t)$ is available.

(A2) The signal $d(t)$ and its derivatives up to the second order are bounded, where the upper bounds can be unknown (at the undifferentiable points, the rightand left-hand derivatives are meant).

\section{The Nonlinear Robust Disturbance Observer}

The proposed nonlinear disturbance observer in this paper is inspired by the sliding mode method.
Based on (1), the following dynamical systems are constructed:

$$
\dot{\hat{x}}(t)=f(x(t), u(t), t)+w(t), \quad \widehat{x}(0)=\widehat{x}_{0},
$$

where $\widehat{x}(t)=\left[\widehat{x}_{1}(t), \ldots, \widehat{x}_{n}(t)\right]^{T} \in R^{n}$ is the reconstructed state which can be obtained by solving $(2) ; \widehat{x}_{0}$ is the initial value of $\widehat{x}(t) ; w(t)=\left[w_{1}(t), \ldots, w_{n}(t)\right]^{T} \in R^{n}$ is a vector which will be determined later.

Inspired by the robustness of sliding mode control technique, we try to find a formulation of $w(t)$ such that $x(t)-$ $\hat{x}(t) \rightarrow 0$ and $\dot{x}(t)-\dot{\hat{x}}(t) \rightarrow 0$ as $t \rightarrow \infty$. The components $w_{i}(t)$ (for $i=1, \ldots, n$ ) are given by the following equations:

$$
\begin{aligned}
\dot{w}_{i}(t)= & -\left(\alpha_{i}+\beta_{i}\right) w_{i}(t)+\widehat{\chi}_{i}(t) \\
& \cdot \operatorname{sign}\left(x_{i}(t)-\widehat{x}_{i}(t)\right)+\alpha_{i} \beta_{i}\left(x_{i}(t)-\widehat{x}_{i}(t)\right),
\end{aligned}
$$

where $\widehat{\chi}_{i}(t)$ are defined as

$$
\widehat{\chi}_{i}(t)=\gamma_{i}\left(\left|x_{i}(t)-\widehat{x}_{i}(t)\right|+\alpha_{i} q_{i}(t)\right)
$$

with

$$
\dot{q}_{i}(t)=\left|x_{i}(t)-\widehat{x}_{i}(t)\right| ;
$$

$\alpha_{i}, \beta_{i}$, and $\gamma_{i}$ are positive constants; $w_{i}(0)$ can be any values; $q_{i}(0)$ should be positive constants.

Theorem 1. For $i=1, \ldots, n$, the generated signals $w_{i}(t)$ and $\hat{\chi}_{i}(t)$ are uniformly bounded; $\left(x_{i}(t)-\widehat{x}_{i}(t)\right)$ $\epsilon L^{1}(0, \infty)$ and $\left(d_{i}(t)-w_{i}(t)\right) \in L^{2}(0, \infty)$. Furthermore, it holds that

$$
\begin{aligned}
& \lim _{t \rightarrow \infty}\left(x_{i}(t)-\widehat{x}_{i}(t)\right)=0, \\
& \lim _{t \rightarrow \infty}\left(d_{i}(t)-w_{i}(t)\right)=0 .
\end{aligned}
$$

Proof. Define

$$
\begin{gathered}
e_{i}(t)=x_{i}(t)-\hat{x}_{i}(t), \\
\sigma_{i}(t)=\dot{e}_{i}(t)+\alpha_{i} e_{i}(t) .
\end{gathered}
$$

Then, differentiating $e_{i}(t)$ and $\sigma_{i}(t)$ yields

$$
\begin{gathered}
\dot{e}_{i}(t)=d_{i}(t)-w_{i}(t), \\
\dot{\sigma}_{i}(t)=\dot{d}_{i}(t)-\dot{w}_{i}(t)+\alpha_{i}\left\{d_{i}(t)-w_{i}(t)\right\} \\
=\eta_{i}(t)-\beta_{i} \sigma_{i}(t)-\widehat{\chi}_{i}(t) \cdot \operatorname{sign}\left(e_{i}(t)\right)
\end{gathered}
$$

with

$$
\eta_{i}(t)=\dot{d}_{i}(t)+\left(\alpha_{i}+\beta_{i}\right) d_{i}(t) .
$$

By Assumption (A2), there exist positive constants $\chi_{i}$ such that

$$
\left|\eta_{i}(t)\right|+\frac{1}{\beta_{i}}\left|\dot{\eta}_{i}(t)\right| \leq \chi_{i}
$$


Consider the Lyapunov candidate

$$
V_{i}(t)=\frac{1}{2} \sigma_{i}^{2}(t)+\frac{1}{2 \gamma_{i}}\left(\widehat{\chi}_{i}(t)-\chi_{i}\right)^{2} .
$$

Differentiating $V_{i}(t)$ yields

$$
\begin{aligned}
\dot{V}_{i}(t)= & \sigma_{i}(t)\left(\eta_{i}(t)-\beta_{i} \sigma_{i}(t)-\widehat{\chi}_{i}(t) \cdot \operatorname{sign}\left(e_{i}(t)\right)\right) \\
& +\left(\widehat{\chi}_{i}(t)-\chi_{i}\right)\left(\frac{d}{d t}\left|e_{i}(t)\right|+\alpha_{i}\left|e_{i}(t)\right|\right) \\
= & -\beta_{i} \sigma_{i}^{2}(t)+\sigma_{i}(t) \eta_{i}(t)-\left(\dot{e}_{i}(t)+\alpha_{i} e_{i}(t)\right) \widehat{\chi}_{i}(t) \\
& \cdot \operatorname{sign}\left(e_{i}(t)\right)+\left(\widehat{\chi}_{i}(t)-\chi_{i}\right)\left(\frac{d}{d t}\left|e_{i}(t)\right|+\alpha_{i}\left|e_{i}(t)\right|\right) \\
= & -\beta_{i} \sigma_{i}^{2}(t)+\sigma_{i}(t) \eta_{i}(t)-\chi_{i} \cdot \frac{d}{d t}\left|e_{i}(t)\right|-\alpha_{i} \chi_{i}\left|e_{i}(t)\right| .
\end{aligned}
$$

Integrating both sides of (15) from 0 to $t$ yields

$$
\begin{aligned}
V_{i}(t)= & V_{i}(0)-\beta_{i} \int_{0}^{t} \sigma_{i}^{2}(\tau) d \tau \\
& +\int_{0}^{t}\left(\dot{e}_{i}(\tau)+\alpha_{i} e_{i}(\tau)\right) \eta_{i}(\tau) d \tau \\
& -\chi_{i} \int_{0}^{t} \frac{d}{d t} e_{i}(\tau) d \tau-\alpha_{1} \chi_{1} \int_{0}^{t}\left|e_{i}(\tau)\right| d \tau \\
= & V_{i}(0)-\beta_{i} \int_{0}^{t} \sigma_{i}^{2}(\tau) d \tau+e_{i}(t) \eta_{i}(t)-e_{i}(0) \eta_{i}(0) \\
& +\int_{0}^{t} e_{i}(\tau)\left(-\dot{\eta}_{i}(\tau)-\alpha_{i} \eta_{i}(\tau)\right) d \tau \\
& -\chi_{i}\left|e_{i}(t)\right|-\alpha_{i} \chi_{i} \int_{0}^{t}\left|e_{i}(\tau)\right| d \tau \\
\leq & V_{i}(0)-\beta_{i} \int_{0}^{t} \sigma_{i}^{2}(\tau) d \tau+\left|e_{i}(t)\right|\left(\eta_{i}(t)-\chi_{i}\right) \\
& +\int_{0}^{t}\left|e_{i}(\tau)\right|\left(\left|\dot{\eta}_{i}(\tau)\right|+\alpha_{i}\left|\eta_{i}(\tau)\right|-\alpha_{i} \chi_{i}\right) d \tau \\
& +\chi_{i}\left|e_{i}(0)\right|-e_{i}(0) \eta_{i}(0) \\
\leq & V_{i}(0)-\beta_{i} \int_{0}^{t} \sigma_{i}^{2}(\tau) d \tau+\chi_{i}\left|e_{i}(0)\right|-e_{i}(0) \eta_{i}(0),
\end{aligned}
$$

where (13) is employed in the last step. Thus, relation (16) implies that $V_{i}(t)$ and $\int_{0}^{t} \sigma_{i}^{2}(\tau) d \tau$ are uniformly bounded (i.e., $\left.\sigma_{i}(t) \in L^{2}(0, \infty)\right)$. Since the uniform boundedness of $V_{i}(t)$ implies the uniform boundedness of $\sigma_{i}(t)$ and $\widehat{\chi}_{i}(t)$, the uniform boundedness of $\dot{\sigma}_{i}(t)$ can be concluded by observing the expression of $\dot{\sigma}_{i}(t)$ in (11) and Assumption (A2). Therefore, by applying Corollary 1.2.2 of Barbalat's Lemma in [21], it yields $\lim _{t \rightarrow \infty} \sigma_{i}(t)=0$ by observing the uniform boundedness of $\dot{\sigma}_{i}(t)$ and $\int_{0}^{t} \sigma_{i}^{2}(\tau) d \tau$.
On the other hand, by (4) and (5), it is obvious that the uniform boundedness of $\widehat{\chi}_{i}(t)$ means $e_{i}(t) \epsilon$ $L^{\infty}(0, \infty)$ and $e_{i}(t) \in L^{1}(0, \infty)$. Thus, from (9), it can be seen that $\dot{e}_{i}(t)=\sigma_{i}(t)-\alpha_{i} e_{i}(t)$ is uniformly bounded. Now, by applying Barbalat's lemma again, it gives $\lim _{t \rightarrow \infty} e_{i}(t)=0$. Then, $\lim _{t \rightarrow \infty} \dot{e}_{i}(t)=\lim _{t \rightarrow \infty}\left(\sigma_{i}(t)-\alpha_{i} e_{i}(t)\right)=0$. Therefore, from (10), relation (7) is proved.

By the fact that $e_{i}(t) \in L^{\infty}(0, \infty) \cap L^{1}(0, \infty)$, it gives $e_{i}(t) \in L^{2}(0, \infty)$ (see [21]). By noticing $\dot{e}_{i}(t)=\sigma_{i}(t)-$ $\alpha_{i} e_{i}(t)$, it yields $\dot{e}_{i}(t) \in L^{2}(0, \infty)$. Therefore, $\left(d_{i}(t)-w_{i}(t)\right) \epsilon$ $L^{2}(0, \infty)$. The theorem is proved.

Remark 2. The upper bounds $\chi_{i}$ in (13) need not be known. They are updated by the adaptive algorithms defined in (4)-(5). The parameters $\alpha_{i}$ and $\gamma_{i}$ should be chosen as large constants so that a fast adjustment of $\widehat{\chi}_{i}(t)$ can be obtained.

Remark 3. $\sigma_{i}(t)=0$ can be regarded as the sliding surface in $\left(e_{i}(t), \dot{e}_{i}(t)\right)$ plane. By the definition of $\sigma_{i}(t)$ in $(9)$, it can be seen that the parameters $\alpha_{i}$ should be chosen to be large enough in order to get fast convergences of $e_{i}(t)$. By observing (16), it can be seen that the parameters $\beta_{i}$ should also be chosen to be large enough in order to get a fast convergence of $\sigma_{i}(t)$.

Remark 4. The initial value $\widehat{x}_{0}$ of $\widehat{x}(t)$ can be chosen to be any value. Certainly, if the initial value $x(0)$ of the state $x(t)$ is known, it is convenient to choose $\widehat{x}_{0}=x(0)$.

\section{Simulation Results}

Consider the system described by

$$
\dot{x}(t)=-0.2 x(t)+d(t),
$$

where $x(t)$ is the state; $d(t)$ is the disturbance.

In simulation study, suppose $x(0)=2$ and the disturbance $d(t)$ is

$$
d(t)=\cos (\pi t)\left(1+\frac{0.8 x^{2}(t)}{|x(t)|+0.5}\right) .
$$

Since system (17) is one dimension, we will omit the subscripts in the following. The parameters are chosen as $\alpha=$ $\beta=\gamma=500$. The initial values are chosen as $\widehat{x}(0)=w(0)=$ $0.5, q(0)=0$.

The computer simulation is conducted by MATLAB, where the sampling period is set to 0.001 seconds. Figure 1 shows the disturbance $d(t)$. The estimation error $d(t)-$ $w(t)$ is shown in Figure 2. It can be seen that the convergence is very fast and the estimation error in the steady state is very small. A much smaller estimation error can be achieved by choosing much larger parameters $\alpha, \beta$, and $\gamma$.

In order to test the robustness to measurement noise, suppose that a measurement noise $v(t)$ is accompanied with the state. In the simulation study, suppose the noise $v(t)$, which is shown in Figure 3, is a band-limited random noise whose sampling period is 0.001 . By using the same design parameters, the disturbance estimation error is given in 


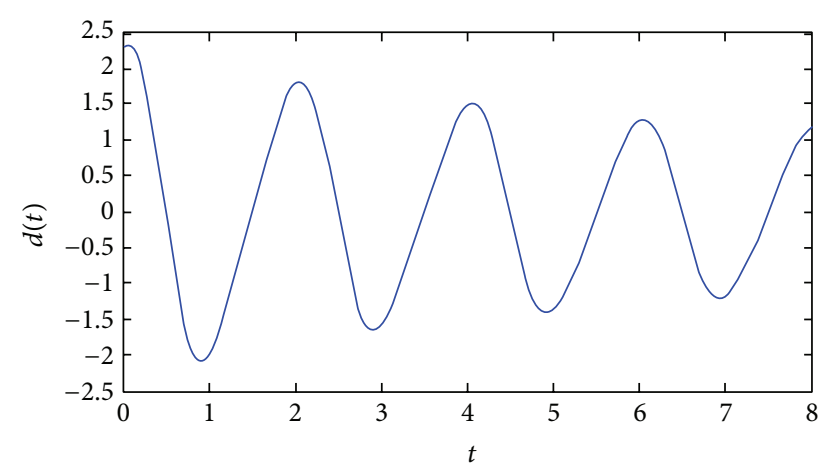

FIgURE 1: The disturbance signal $d(t)$.

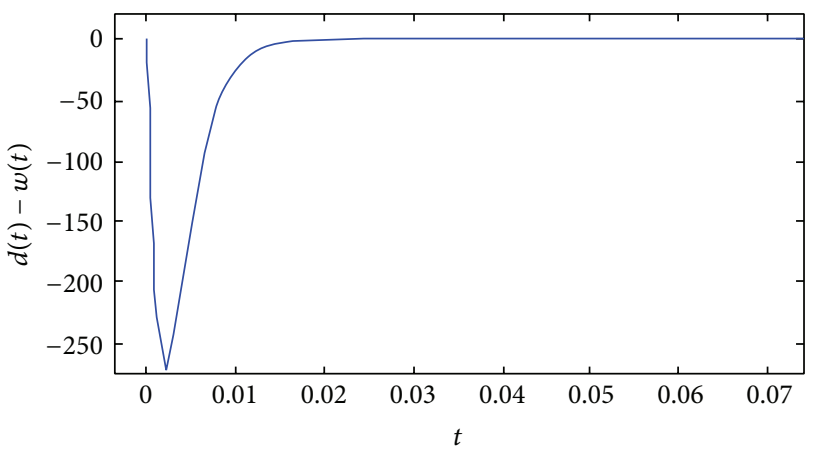

(a) The estimation error as $t$ is small

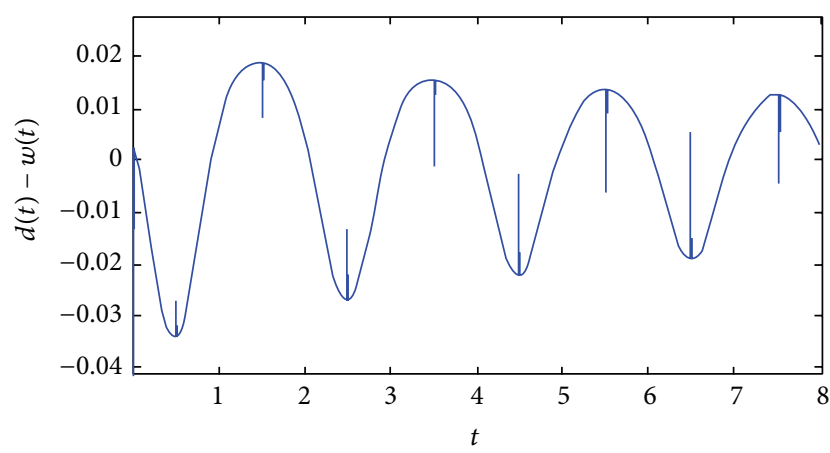

(b) The estimation error at steady state

Figure 2: The estimation error $d(t)-w(t)$.

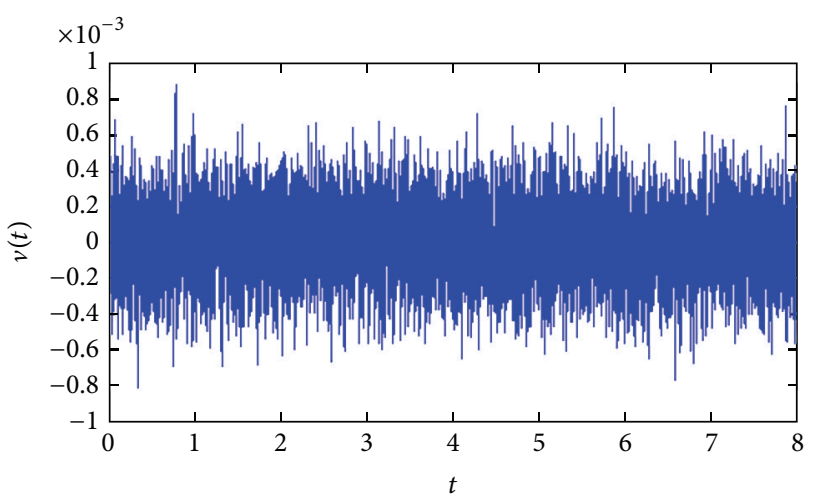

Figure 3: The measurement noise $v(t)$.

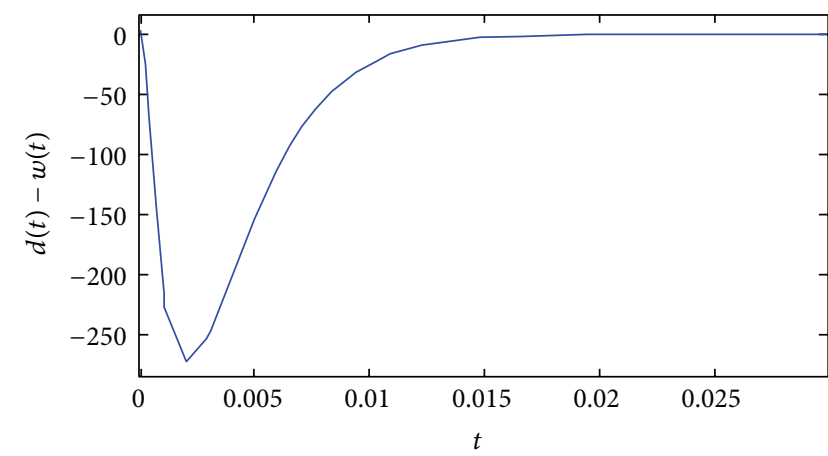

(a) The estimation error as $t$ is small

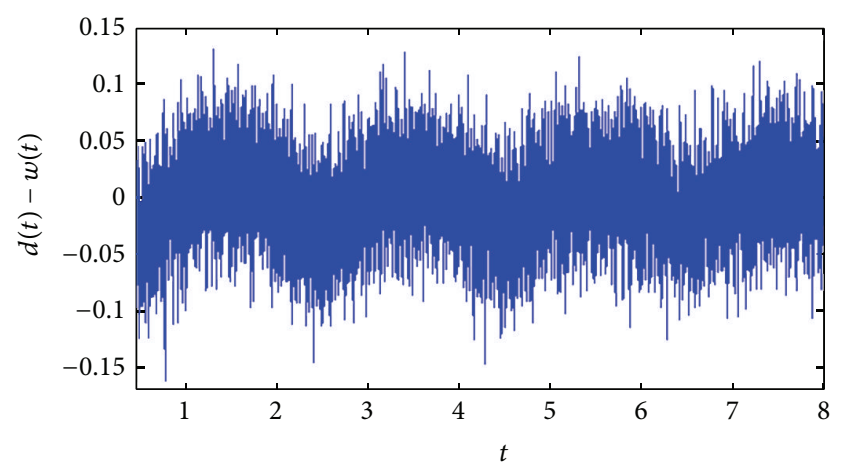

(b) The estimation error at steady state

Figure 4: The estimation error $d(t)-w(t)$ when measurement noise exists.

Figure 4. Due to the existence of the measurement noises, the estimation error becomes larger.

Now, let us compare the proposed method with the formulation in [19]. The disturbance observer is given by

$$
\begin{array}{r}
\dot{\hat{\widehat{x}}}(t)+\lambda \widehat{x}(t)=(\lambda-0.2) x(t)+m(t), \\
\widehat{\widehat{x}}(0)=0.5,
\end{array}
$$

where $m(t)$ is determined by

$$
m(t)=\frac{(x(t)-\widehat{\widehat{x}}(t)) \widehat{C}(t)}{|x(t)-\widehat{\widehat{x}}(t)|+\delta} ;
$$

$\widehat{C}(t)$ is updated by

$$
\begin{aligned}
& \dot{\vec{C}}(t)= \begin{cases}\kappa|x(t)-\widehat{\hat{x}}(t)| & \text { if }|x(t)-\widehat{\hat{x}}(t)|>\sqrt{\frac{2 \delta \widehat{C}(t)}{\lambda}} \\
0 & \text { otherwise, }\end{cases} \\
& \widehat{C}(0)=0.5 \text {. }
\end{aligned}
$$

In order to get a similar estimation error in the steady state as shown in Figure 2, the parameters are chosen as $\lambda=2, \kappa=5$, and $\delta=0.006$. The estimation error $d(t)-m(t)$ is shown in Figure 5. It can be seen that transient time of the new observer 


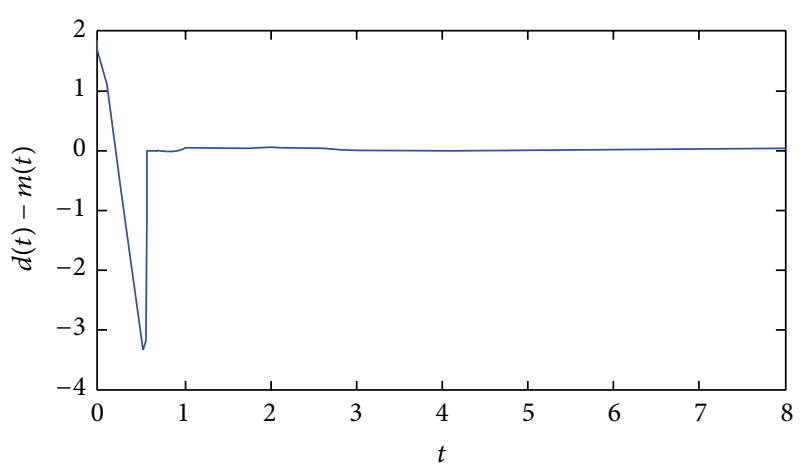

(a) The estimation error for $0 \leq t \leq 8$

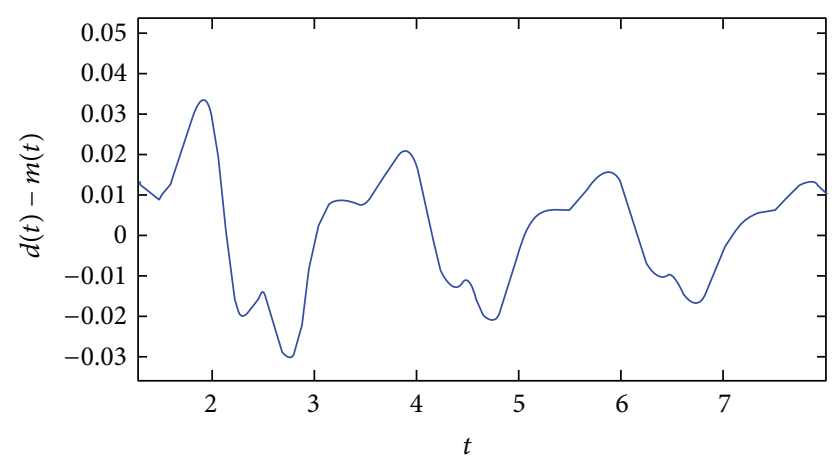

(b) The estimation error at steady state

FIGURE 5: The estimation error $d(t)-m(t)$ by using the observer in [19].

is much shorter than that of the observer in [19] in order to get the same estimation error.

Now, let us investigate the robustness to measurement noises of the observer in [19]. Suppose the same measurement noise which is shown in Figure 3 exists in the state. By using the same design parameters, the disturbance estimation error is shown in Figure 6. It can be seen that the estimation error becomes much larger than that in Figure 4. By comparing Figures 4 and 6 , it can be seen that the new disturbance observer is much more robust to the measurement noises.

\section{Conclusions}

In this paper, inspired by the sliding mode control theory, a nonlinear exact disturbance observer is proposed based on state space approach. The disturbance and its derivatives up to the second order are assumed to be bounded. In the disturbance estimation, the bounds relating to the disturbances are simultaneously updated online by an adaptive algorithm. The exact estimation of the disturbance can be achieved theoretically. The convergence speed of the disturbance estimation error is controlled by design parameters. The proposed method is robust to the type of disturbance and is easy to be implemented. Computer simulation results show that the proposed method is effective and is superior to the method in [19] in the sense of transient time and robustness to measurement noises. The proposed observer is expected to be developed for the systems in transfer function domain.

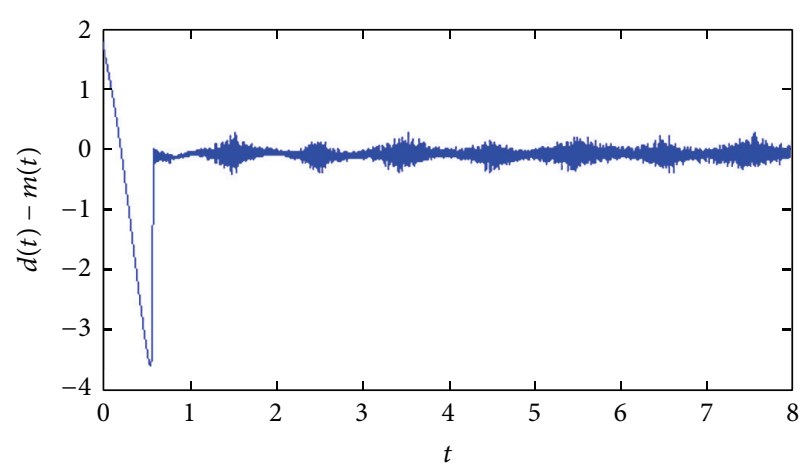

(a) The estimation error for $0 \leq t \leq 8$

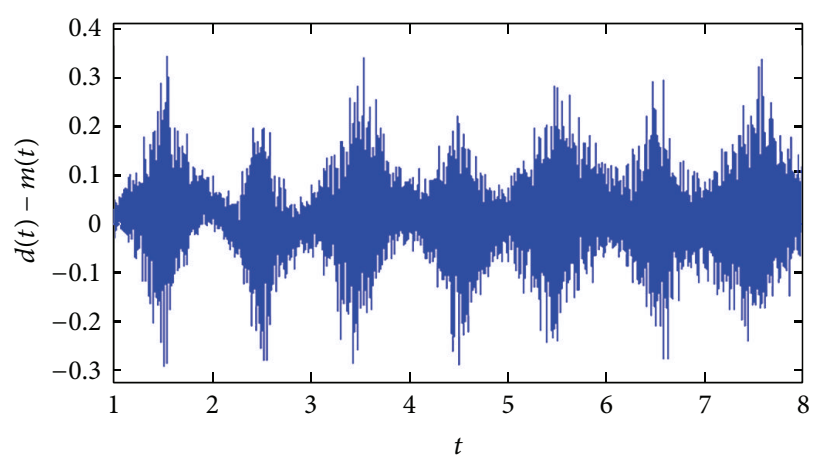

(b) The estimation error at steady state

FIGURE 6: The estimation error $d(t)-m(t)$ by using the observer in [19] when measurement noise exists.

\section{Conflict of Interests}

The author declares that there is no conflict of interests regarding the publication of this paper.

\section{References}

[1] X. Chen, S. Komada, and T. Fukuda, "Design of a nonlinear disturbance observer," IEEE Transactions on Industrial Electronics, vol. 47, no. 2, pp. 429-437, 2000.

[2] J. Qiao and L. Guo, "Antidisturbance fault tolerant control of attitude control systems for microsatellite with unknown input delay," Mathematical Problems in Engineering, vol. 2013, Article ID 804754, 9 pages, 2013.

[3] Y. Zhou and M.-L. Chen, "Sliding mode control for NSVs with input constraint using neural network and disturbance observer," Mathematical Problems in Engineering, vol. 2013, Article ID 904830, 12 pages, 2013.

[4] H. Sun and L. Hou, "Composite disturbance observer-based control and $H_{\infty}$ output tracking control for discrete-time switched systems with time-varying delay"' Mathematical Problems in Engineering, vol. 2013, Article ID 698935, 12 pages, 2013.

[5] Y. M. T. Elobaid, J. Huang, and Y. Wang, "Nonlinear disturbance observer based robust tracking control of pneumatic muscle," Mathematical Problems in Engineering, vol. 2014, Article ID 872093, 8 pages, 2014.

[6] X. Lei, K. Guo, and S. S. Ge, "Disturbance observer based control of small unmanned aerial rotorcraft," Mathematical 
Problems in Engineering, vol. 2013, Article ID 464938, 9 pages, 2013.

[7] T. Umeno and Y. Hori, "Robust speed control of DC servomotors using modern two degrees-of-freedom controller design," IEEE Transactions on Industrial Electronics, vol. 38, no. 5, pp. 363-368, 1991.

[8] T. Umeno, T. Kaneko, and Y. Hori, "Robust servosystem design with two degrees of freedom and its application of novel motion control of robot manipulators," IEEE Transactions on Industrial Electronics, vol. 40, no. 5, pp. 473-485, 1993.

[9] Q.-C. Zhong and J. E. Normey-Rico, "Control of integral processes with dead-time. Part 1: disturbance observer-based 2DOF control scheme," IEE Proceedings: Control Theory and Applications, vol. 149, no. 4, pp. 285-290, 2002.

[10] K. Yamada, S. Komada, M. Ishida et al., "Characteristic of servo system using high order disturbance observer," in Proceedings of the 35th Conference on Decision and Control, pp. 3252-3257, Kobe, Japan, December 1996.

[11] W.-H. Chen, "Disturbance observer based control for nonlinear systems," IEEE/ASME Transactions on Mechatronics, vol. 9, no. 4, pp. 706-710, 2004.

[12] C. Edwards and S. K. Spurgeon, "On the development of discontinuous observers," International Journal of Control, vol. 59, no. 5, pp. 1211-1229, 1994.

[13] K.-S. Kim, K.-H. Rew, and S. Kim, "Disturbance observer for estimating higher order disturbances in time series expansion," IEEE Transactions on Automatic Control, vol. 55, no. 8, pp. 19051911, 2010.

[14] A. E. Rundell, S. V. Drakunov, and R. A. DeCarlo, "A sliding mode observer and controller for stabilization of rotational motion of a vertical shaft magnetic bearing," IEEE Transactions on Control Systems Technology, vol. 4, no. 5, pp. 598-608, 1996.

[15] B. L. Walcott and S. H. Zak, "Combined observer-controller synthesis for uncertain dynamical systems with applications," IEEE Transactions on Systems, Man and Cybernetics, vol. 18, no. 1, pp. 88-104, 1988.

[16] J. J. Slotine, Applied Nonlinear Control, Prentice Hall, Englewood Cliffs, NY, USA, 1991.

[17] V. I. Utkin, Sliding Modes in Control Optimization, Springer, New York, NY, USA, 1992.

[18] K. D. Young, V. I. Utkin, and Ü. Özgüner, "A control engineer's guide to sliding mode control," IEEE Transactions on Control Systems Technology, vol. 7, no. 3, pp. 328-342, 1999.

[19] X. Chen, T. Fukuda, and K. D. Young, "A new nonlinear robust disturbance observer," Systems \& Control Letters, vol. 41, no. 3, pp. 189-199, 2000.

[20] X. Chen, C.-Y. Su, and T. Fukuda, "A nonlinear disturbance observer for multivariable systems and its application to magnetic bearing systems," IEEE Transactions on Control Systems Technology, vol. 12, no. 4, pp. 569-577, 2004.

[21] S. Sastry and M. Bodson, Adaptive Control, Stability, Convergence, and Robustness, Prentice Hall, Englewood Cliffs, NJ, USA, 1989. 


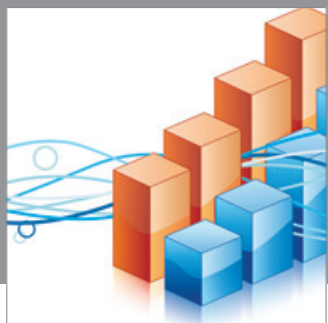

Advances in

Operations Research

mansans

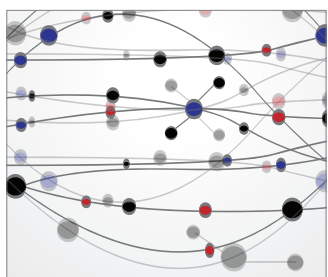

The Scientific World Journal
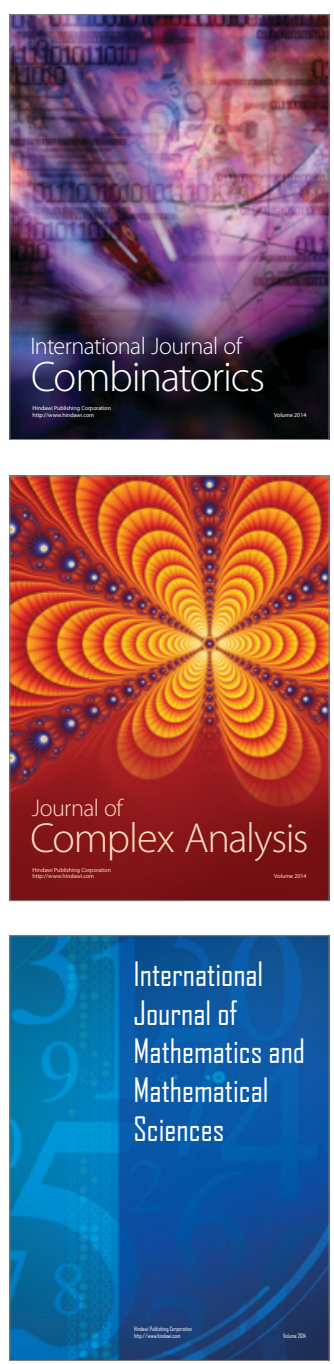
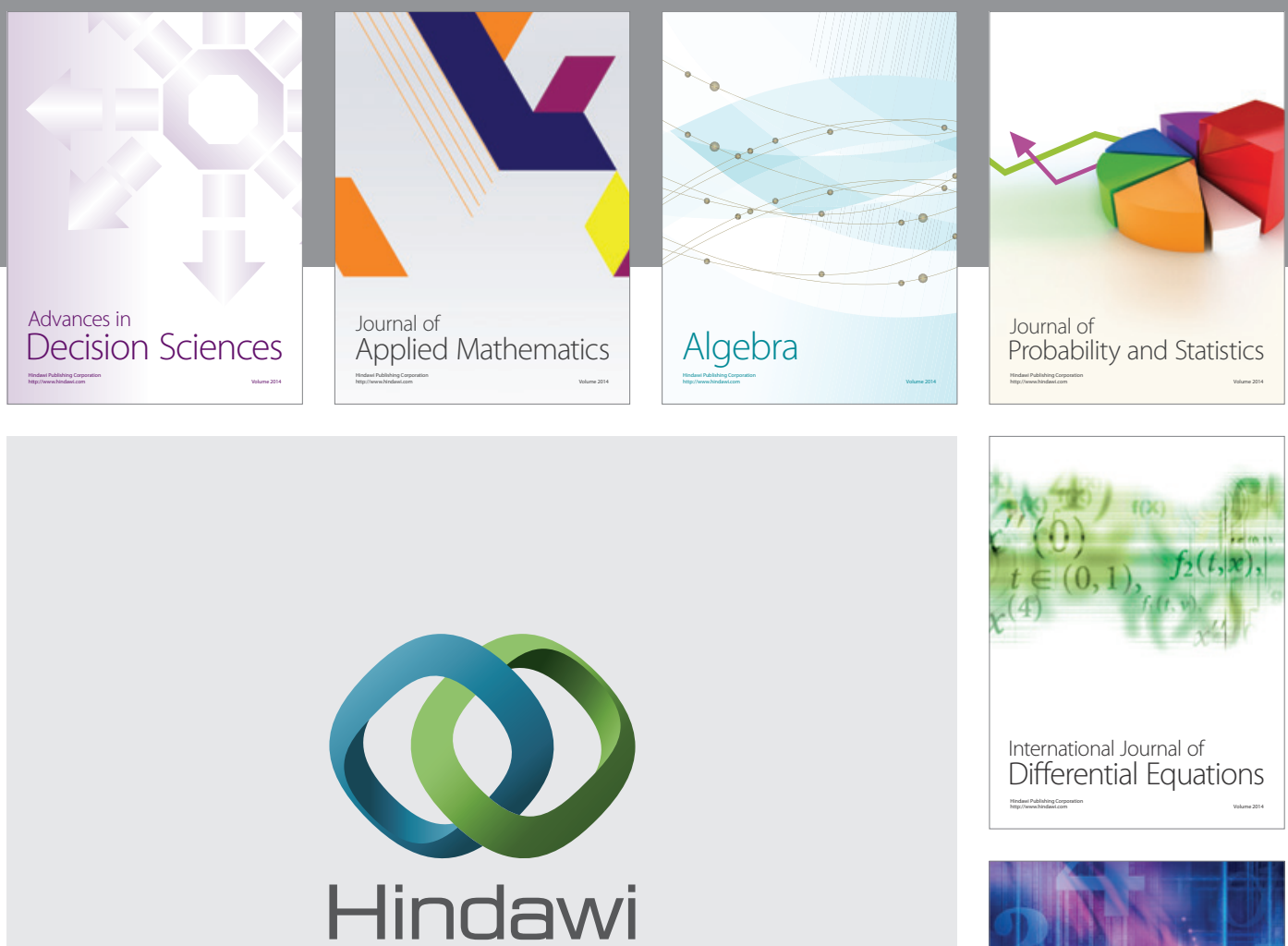

Submit your manuscripts at http://www.hindawi.com
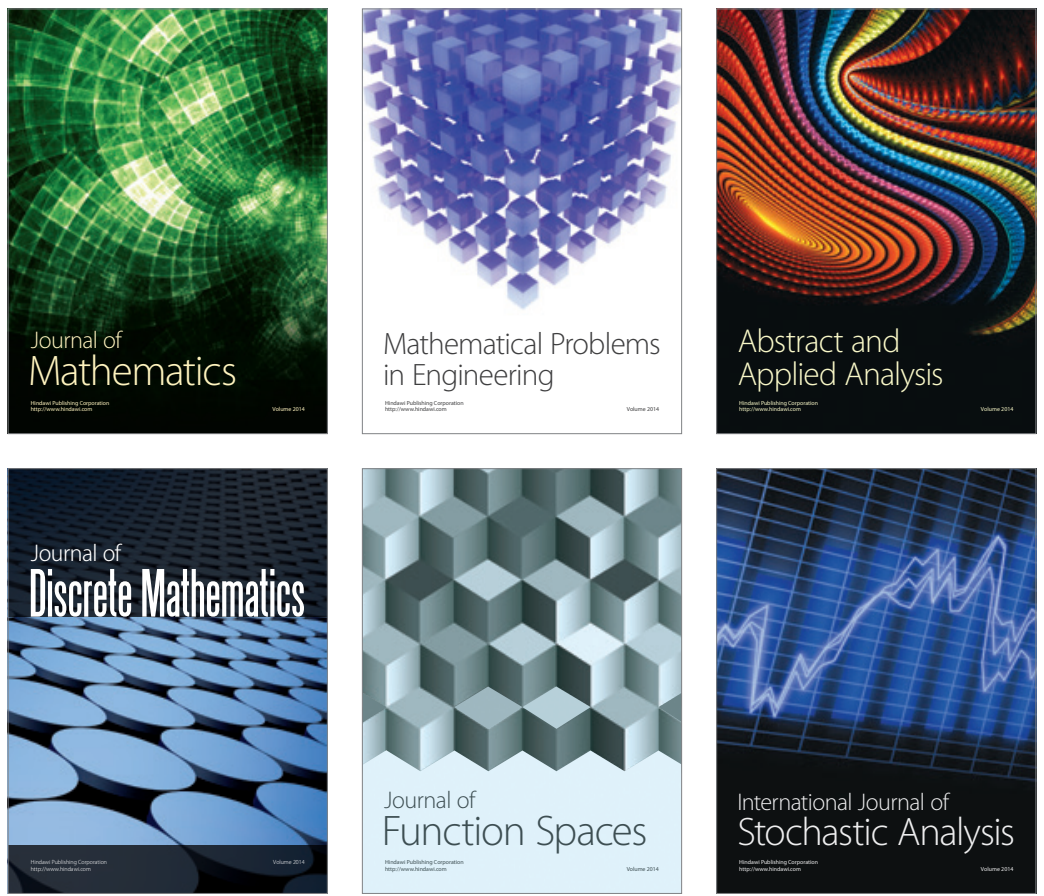

Journal of

Function Spaces

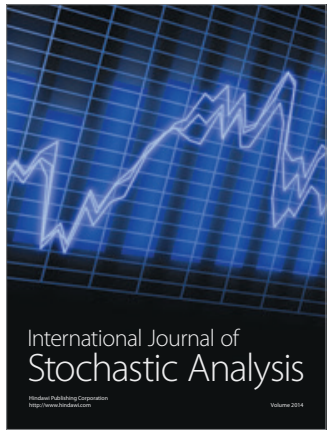

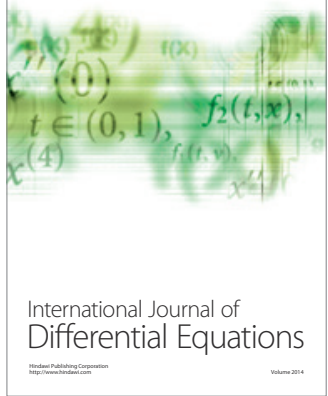
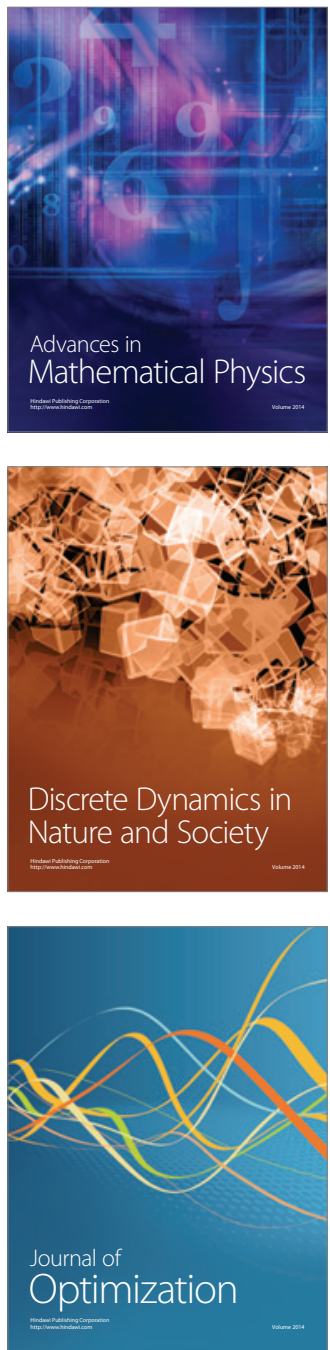